\title{
A study of the magnetic helium variable emission-line star HD 125823.
}

\author{
S. Hubrig ${ }^{1}$, N. Nesvacil ${ }^{2}$, F. González ${ }^{3}$, B. Wolff ${ }^{4}$, I. Savanov ${ }^{5}$ \\ 1 European Southern Observatory, Casilla 19001, Santiago, Chile \\ shubrig@eso.org, \\ 2 Institut für Astronomie, Universität Wien, Türkenschanzstr. 17, 1180 Vienna, \\ Austria nicole@jan.astro.univie.ac.at, \\ 3 Complejo Astronómico El Leoncito, Casilla 467, 5400 San Juan, Argentina \\ fgonzalez@casleo.gov.ar, \\ 4 European Southern Observatory, Karl-Schwarzschild-Str. 2, 85748 Garching, \\ Germany bwolff@eso.org, \\ 5 Astrophysikalisches Institut Potsdam, An der Sternwarte 16, 14482 Potsdam, \\ Germany isavanov@aip.de
}

The $5.9 \mathrm{M}_{\odot}$ star HD 125823 is a striking helium variable with a period of $8.82 \mathrm{~d}$, ranging in helium spectral type from He-strong B2 to B8 (e.g., Norris 1968 [3]). In fact, HD 125823 seems to be a transition object between the He-weak and He-strong stars with unreddened colors just at the boundary between the two groups in the UBV color-color diagram. Although highdispersion studies have been carried out in the past by several authors (e.g. Wolff \& Morrison 1974 [ ]), no abundance analysis is available.

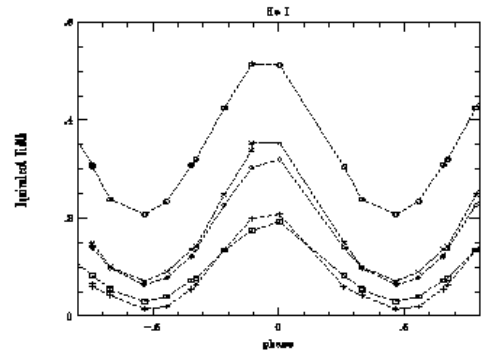

Fig. 1. Variations of the equivalent widths of the He I lines $\lambda \lambda 4121,4438,4713$, 5047 , and 6678 over the rotation period.

Recently, we obtained high spectral resolution high signal-to-noise UVES spectra of HD 125823 over the rotation period of $8.82 \mathrm{~d}$ in order to study the surface distribution of various chemical elements. In Fig. 1 we show the behaviour of the He I lines $\lambda \lambda 4121,4438,4713,5047$, and 6678 over the rotation period. The magnetic field observations reported in the literature (Wolff \& Morrison 1974 [6]; Borra et al. 1983 [1]) indicate that the negative extremum coincides closely in phase with the maximum helium strength. 


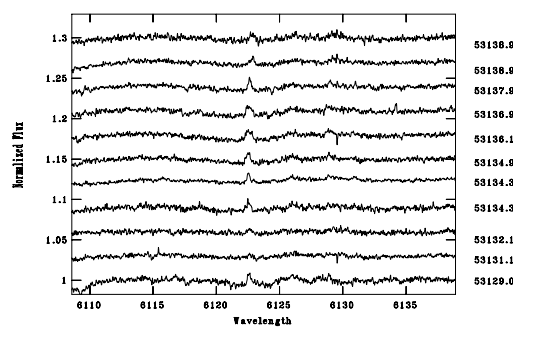

Fig. 2. Mn II emission lines of multiplet 13 in the spectra of HD 125823 obtained on nine consecutive nights with UVES.

However, the positive magnetic pole shows a He-deficient cap, so that the two magnetic poles are associated with very different helium abundances.

An additional interest to study HD 125823 comes from the recent detection of weak emission lines of various ions (Mn II, Fe II, Cr II, Ti II, etc.) in optical spectra of B-type stars (e.g., Sigut et al. 2000 [4; Wahlgren \& Hubrig 2000 [5] Castelli \& Hubrig 2004 [2]). For the first time we present here observational evidence for the appearance of emission lines of $\mathrm{Mn}$ and Fe in a variable magnetic star. In Fig. 2 we show the behaviour of the emission line profiles of the Mn II multiplet 13. It is remarkable that the emission lines of Mn II are definitely variable, changing their appearance from the first night to the last. To date, explanations of this phenomenon have been put forward in the context of non-LTE line formation and possible fluorescence mechanisms. The qualitative and quantitative assessments of the spectra of B type stars suggest a possible correlation of the appearance of diverse emission lines with the non-magnetic $\mathrm{P}-\mathrm{Ga}$ and $\mathrm{HgMn}$ groups as well as with spectral type.

From the behaviour of the line profile variations of various elements we can conclude that helium is enhanced in regions of the stellar surface where silicon and other metals are depleted, and helium is depleted in regions where the metals are enhanced. A future goal is to derive surface abundance maps for various elements. Such a study is an important step towards understanding the effect of magnetic fields on the development of surface chemical peculiarities in the photospheres of hot stars.

\section{References}

1. Borra E. F., Landstreet J. D., Thompson I., 1983, ApJS, 53, 151

2. Castelli F., Hubrig S., 2004, A\&A, 425, 263

3. Norris J., 1968, Natur, 219, 1342

4. Sigut T. A. A., Landstreet J. D., Shorlin S. L. S., 2000, ApJ, 530, L89

5. Wahlgren G. M., Hubrig S., 2000, A\&A, 362, L13

6. Wolff S. C., Morrison N. D., 1974, PASP, 86, 935 